\title{
The effect of transmission- line dynamics on a globally synchronizing controller for power inverters
}

\section{Conference Paper}

Author(s):

Brouillon, Jean-Sébastien; Colombino, Marcello; Gross, Dominic (D); Dörfler, Florian (1)

Publication date:

2018

Permanent link:

https://doi.org/10.3929/ethz-b-000346278

Rights / license:

In Copyright - Non-Commercial Use Permitted

Originally published in:

https://doi.org/10.23919/ECC.2018.8550600

Funding acknowledgement:

691800 - Massive InteGRATion of power Electronic devices (SBFI) 


\title{
The effect of transmission-line dynamics on a globally synchronizing controller for power inverters
}

\author{
Jean-Sébastien Brouillon, Marcello Colombino, Dominic Groß and Florian Dörfler
}

\begin{abstract}
In this work, we analyze a dispatchable virtual oscillator control (dVOC) strategy for grid-forming power inverters that ensures almost global synchronization of inverterbased AC power systems when the dynamics of the transmission network are neglected, i.e., if an algebraic model of the transmission lines is used. While this approximation is often justified for conventional power systems, the dynamics of the transmission lines can compromise the stability of an inverterbased power system. Therefore, in this article, we use tools from singular perturbation theory to construct a Lyapunov function candidate and explicit bounds on the controller gains that guarantee global convergence to the set of steady-states for the full power-system with transmission line dynamics. Moreover, we show that the only undesirable steady-state is unstable.
\end{abstract}

\section{INTRODUCTION}

The electric power grid is undergoing a period of unprecedented change. A major transition is the replacement of bulk generation based on synchronous machines by distributed generation interconnected to the grid via power electronics devices fed by renewable energy sources. This gives rise to scenarios in which either parts of the transmission grid or an islanded distribution grid may operate without conventional synchronous generation. In either case, the power grid faces great challenges due to the loss of rotational inertia and the self-synchronizing dynamics of synchronous machines.

The problem of synchronization has been widely studied in the recent literature. Most of the common approaches study droop control [1], [2]. Other popular approaches rely virtual oscillator control (VOC) [3]-[7]. However, standard VOC cannot be dispatched and track pre-specified power and voltage setpoints. To address this challenge, the authors recently proposed a dispatchable virtual oscillator control (dVOC) strategy [8], [9] with almost global stability guarantees.

In order to simplify the nonlinear stability analysis, the dynamic nature of transmission lines is often neglected in the study of power system transient stability and synchronization. In most of the aforementioned studies, the network of transmission lines is modeled as an admittance matrix that provides an algebraic relationship between voltages and currents. This approximation is commonly used in the analysis of power systems, for instance in the form of the well-known phasor approximation, and can be made rigorous

This work was partially funded by the European Union's Horizon 2020 research and innovation programme under grant agreement $\mathrm{N}^{\circ} 691800$. This article reflects only the authors' views and the European Commission is not responsible for any use that may be made of the information it contains.

J-S Brouillon, D. Groß, and F. Dörfler, are with the Automatic Control Laboratory, ETH Zürich, Switzerland, M. Colombino is with the National Renewable Energy Laboratory (NREL), Golden, CO, USA; email:\{grodo,jeanb,dorfler\}@ethz.ch, marcello.colombino@nrel.gov using time-scale separation arguments [10]. This approximation is justified in a traditional power network, where the bulk generation is provided by synchronous machines with very slow time constants (seconds). As power inverters can be controlled at much faster time-scales (milliseconds), the transmission line dynamics can compromise the stability of the power-network. This phenomenon has been noted in [2], [11] for a droop controlled micro-grids and can be verified experimentally for all control methods listed above. In [12] small signal stability analysis for a steady state with no power flow is used to obtain insightful stability conditions for droop control in combination with a dynamic line model.

In this work we study the dVOC proposed in [8]. In [9] it is shown that $\mathrm{dVOC}$ renders power inverters interconnected through an algebraic network model almost globally asymptotically stable with respect to a synchronous steady-states that corresponds to a desired solution of the power-flow equations. We stress that these stability guarantees are not restricted to operating points with zero relative angles (i.e., zero power flow). The aim of this paper is to provide a first step towards establishing the same stability guarantees when including line dynamics ${ }^{1}$. To this end, we use tools from singular perturbation theory to construct a Lyapunov function and explicit bounds on the controller gains that guarantees convergence of the full power-system with transmission line dynamics. Moreover, we show that the only undesirable steady-state of the system is unstable.

\section{Notation}

The set of real numbers is denoted by $\mathbb{R}, \mathbb{R}_{\geq 0}$ denotes the set of nonnegative reals. For column vectors $x \in \mathbb{R}^{n}$ and $y \in \mathbb{R}^{m}$ we use $(x, y)=\left[x^{\top}, y^{\top}\right]^{\top} \in \mathbb{R}^{n+m}$ to denote a stacked vector, $I_{n}$ denotes the identity matrix of dimension $n$, $\otimes$ denotes the Kronecker product, $\|x\|$ denotes the Euclidean norm, and $\|x\|_{C}:=\min _{z \in \mathcal{C}}\|z-x\|$ denotes the distance of a point $x$ to a set $\mathcal{C}$. Given $\theta \in[0,2 \pi]$ the $2 \mathrm{D}$ Rotation matrix is denoted by $R(\theta)$, and we define $J:=R(\pi / 2)$ and $\mathcal{J}_{n}:=I_{n} \otimes J$. Given a matrix $A, A^{\top}$ denotes its transpose, $\|A\|$ denotes its induced 2-norm, and $A \succ 0$ denotes that $A$ is positive definite. Moreover, we use $\varphi_{f}\left(t, x_{0}\right)$ to denote the solution of $\frac{\mathrm{d}}{\mathrm{d} t} x=f(x)$ at time $t \geq 0$ starting from the initial condition $x(0)=x_{0}$ at time $t_{0}=0$.

\section{MODELING AND CONTROL OF AN INVERTER BASED POWER NETWORK}

In this section, we introduce the model of the inverterbased power grid that will be studied throughout the paper.

\footnotetext{
${ }^{1}$ Stability conditions that ensure global asymptotic stability including line dynamics can be found in the preprint [13].
} 


\section{A. Inverter-based power grid}

We study the control of $N$ three-phase inverters interconnected by $M$ resistive-inductive transmission lines, i.e., it is assumed that the shunt admittances of the transmission lines can be neglected. All electrical quantities in the network are assumed to be balanced. This allows us to work in $\alpha \beta$ coordinates, which we obtain by applying the well-known Clarke transformation to the three-phase variables [14]. To each inverter we associate a terminal voltage vector $\underline{v}_{k} \in \mathbb{R}^{2}$, that can be fully controlled, and an output current $\underline{i}_{o, k} \in$ $\mathbb{R}^{2}$ flowing out of the inverter and into the network. We model the transmission network as a simple, undirected, and weighted graph $\mathcal{G}=(\mathcal{N}, \mathcal{E}, \mathcal{W})$, where $\mathcal{N}=\{1, \ldots, N\}$ is the set of nodes corresponding to the inverters, $\mathcal{E} \subseteq \mathcal{N} \times N$, with $|\mathcal{E}|=M$, is the set of undirected edges corresponding to the transmission lines. To each transmission line, we associate an impedance matrix $Z_{l}:=I_{2} r_{l}+\omega_{0} J \ell_{l}$ and an admittance matrix $Y_{l}:=Z_{l}^{-1}$ with

$$
\left\|Y_{l}\right\|=\frac{1}{\sqrt{r_{l}^{2}+\omega_{0}^{2} \ell_{l}^{2}}}
$$

where $r_{l} \in \mathbb{R}_{>0}$ and $\ell_{l} \in \mathbb{R}_{>0}$ are the resistance and inductance of the line $l \in\{1, \ldots, M\}$ respectively, and $\omega_{0} \in \mathbb{R}_{\geq 0}$ is the nominal operating frequency of the power system. The oriented incidence matrix of the graph is denoted by $B$ and, duplicating each edge for the $\alpha$ and $\beta$ components, we define $\mathcal{B}:=B \otimes I_{2}$. The graph Laplacian matrix is defined as $L:=B \operatorname{diag}\left(\left\{\left\|Y_{l}\right\|\right\}_{l=1}^{M}\right) B^{\top}$, where $\left\|Y_{l}\right\|$ is the weight associated with the transmission line with index $l \in\{1, \ldots, M\}$. Moreover, we define the extended Laplacian $\mathcal{L}:=L \otimes I_{2}$, the resistance matrix $R_{T}:=\operatorname{diag}\left(\left\{r_{l}\right\}_{l=1}^{M}\right) \otimes$ $I_{2}$, the inductance matrix $L_{T}:=\operatorname{diag}\left(\left\{\ell_{l}\right\}_{l=1}^{M}\right) \otimes I_{2}$, the impedance matrix $Z_{T}:=\operatorname{diag}\left(\left\{Z_{l}\right\}_{l=1}^{M}\right)$, and the steadystate admittance matrix of the network $\mathcal{Y}:=\mathcal{B} Z_{T}^{-1} \mathcal{B}^{\top}$. The network couples the terminal voltages of the inverters $\underline{v}:=\left(\underline{v}_{1}, \ldots, \underline{v}_{N}\right)$ and line currents $\underline{i}:=\left(\underline{i}_{1}, \ldots, \underline{i}_{M}\right)$ as follows

$$
L_{T} \frac{\mathrm{d}}{\mathrm{d} t} \underline{i}=-R_{T} \underline{i}+\mathcal{B}^{\top} \underline{v} .
$$

We also define the vector of inverter output currents as $\underline{i}_{O}:=$ $\left(\underline{i}_{o, 1}, \ldots, \underline{i}_{o, N}\right)=\mathcal{B} \underline{i}$. We require the following standing assumption which is typically satisfied for transmission lines at the same voltage level.

Assumption 1 (Uniform inductance-resistance ratio) The ratio between the inductance and resistance of every transmission line is constant, i.e., for all $(j, k) \in \mathcal{E}$ it holds that $\frac{\ell_{j k}}{r_{j k}}=\rho \in \mathbb{R}_{>0}$.

To simplify the analysis, it is convenient to perform the following change of variables to a rotating reference frame.

$$
v=\operatorname{diag}\left(\mathcal{R}\left(\omega_{0} t\right)\right) \underline{v}, \quad i=\operatorname{diag}\left(I_{M} \otimes R(\theta)\right) \underline{i}
$$

where $\mathcal{R}(\theta)=I_{N} \otimes R(\theta)$. The dynamics (2) become

$$
L_{T} \frac{\mathrm{d}}{\mathrm{d} t} i=-Z_{T} i+\mathcal{B}^{\top} v \text {. }
$$

Using Assumption 1, we obtain $L_{T}^{-1} R_{T}=\rho^{-1} I_{2 M}$ and the steady-state map $i^{s}(v)$ of the line dynamics (4) is given by

$$
i^{s}(v):=\left(\rho^{-1} I_{2 M}+\mathcal{J}_{M} \omega_{0}\right)^{-1} L_{T}^{-1} \mathcal{B}^{\top} v .
$$

\section{B. Control objectives}

The challenge of controlling an inverter based power system is that each controller can only rely on local measurements of the voltages and currents, while the control objectives are global in nature. In particular, we would like to design a decentralized controller of the form

$$
\frac{\mathrm{d}}{\mathrm{d} t} \underline{v}_{k}=u_{k}\left(\underline{v}_{k}, \underline{i}_{o, k}\right) \text {, }
$$

such that the voltages $\underline{v}$ and currents $\underline{i}$ converge to the following steady-state behavior.

- Synchronous frequency: Given a desired synchronous frequency $\omega_{0} \geq 0$, at steady-state it holds that:

$$
\frac{\mathrm{d}}{\mathrm{d} t} \underline{v}_{k}(t)-\omega_{0} J \underline{v}_{k}(t)=0, \quad k \in \mathcal{N},
$$

- Voltage magnitude: Given a voltage magnitude $v^{\star}>0$, at steady-state it holds that:

$$
\left\|\underline{v}_{k}(t)\right\|=v^{\star}, \quad k \in \mathcal{N}
$$

- Steady-state currents: it holds that: $\underline{i}=i^{s}(\underline{v})$,

- Power injection: Given set points $p_{k}^{\star}$ and $q_{k}^{\star}$ for the active and reactive power injection of each inverter, at steady-state it holds that:

$$
\underline{v}_{k}^{\top} \underline{i}_{o, k}=p_{k}^{\star}, \quad \underline{v}_{k}^{\top} J \underline{i}_{o, k}=q_{k}^{\star}, k \in \mathcal{N} .
$$

Using $\underline{i}=i^{s}(\underline{v})$, the local nonlinear specification (9) on the power injection can be equivalently expressed as non-local linear specification on the voltages $\underline{v}$ as follows (see [8, Sec. IV]):

- Phase locking: Given steady-state angles $\theta_{k 1}^{\star}$, at steadystate it holds that:

$$
\underline{v}_{k}(t)=R\left(\theta_{k 1}^{\star}\right) \underline{v}_{1}(t), \quad k \in \mathcal{N} \backslash\{1\} .
$$

Equation (7) and (8) specify that at steady-state, all voltages in the power network evolve as perfectly sinusoidal signals with frequency $\omega_{0}$ and magnitude $v^{\star}$. Equations (10) and (9) fix the relative voltage angles and the power injection at every node. Note, that the set-points $p_{k}^{\star}$ and $q_{k}^{\star}$ need to satisfy the power-flow equations [8]. For ease of presentation all desired voltage magnitudes are assumed to be equal. The results can be extended to individual voltage set-points (see [9], [13]).

\section{Controller and closed loop design}

For every inverter with index $k \in \mathcal{N}$, we propose the decentralized dispatchable virtual oscillator control (dVOC):

$$
u_{k}:=\omega_{0} J \underline{v}_{k}+\eta\left[K_{k} \underline{v}_{k}-R(\kappa) \underline{i}_{o, k}+\alpha \Phi_{k}\left(\underline{v}_{k}\right) \underline{v}_{k}\right],
$$

with gains $\eta>0, \alpha>0$, and

$$
K_{k}:=\frac{1}{v^{\star 2}} R(\kappa)\left[\begin{array}{cc}
p_{k}^{\star} & q_{k}^{\star} \\
-q_{k}^{\star} & p_{k}^{\star}
\end{array}\right], \quad \Phi_{k}\left(\underline{v}_{k}\right):=\frac{v^{\star 2}-\left\|\underline{v}_{k}\right\|^{2}}{v^{\star 2}} I_{2} .
$$

Note that the voltage error $\Phi_{k}$ has been modified compared to [8], [9]. The matrix $K_{k}$ can be equivalently expressed in 
terms of the steady-state angles $\theta_{j k}^{\star}$ and admittance $\left\|Y_{j k}\right\|$ of the line connecting node $j$ and node $k$ as follows (see [8])

$$
K_{k}=\sum_{j:(j, k) \in \mathcal{E}}\left\|Y_{j k}\right\|\left(I_{2}-R\left(\theta_{j k}^{\star}\right)\right), \quad \forall k \in \mathcal{N} .
$$

Using $\Phi(\underline{v}):=\operatorname{diag}\left(\left\{\Phi_{k}\left(\underline{v}_{k}\right)\right\}_{k=1}^{N}\right)$ and $\mathcal{K}:=\operatorname{diag}\left(\left\{K_{k}\right\}_{k=1}^{N}\right)$, the controller (11), together with (2), results in the following closed loop equations for the power network

$$
\begin{aligned}
\frac{\mathrm{d}}{\mathrm{d} t} \underline{v} & =\omega_{0} \mathcal{J}_{N} \underline{v}+\eta[\mathcal{K} \underline{v}-\mathcal{R}(\kappa) \mathcal{B} \underline{i}+\alpha \Phi(\underline{v}) \underline{v}], \\
L_{T} \frac{\mathrm{d}}{\mathrm{d} t} \underline{i} & =-R_{T} \underline{i}+\mathcal{B}^{\top} \underline{v} .
\end{aligned}
$$

\section{A LYAPUNOV CHARACTERIZATION OF ATTRACTIVITY WITH RESPECT TO A SET}

In order to state the main result of the paper, we require the following definitions of global attractivity with respect to a set and its characterization via Lyapunov functions.

Definition 1 (Attractivity with respect to a set) A dynamic system $\frac{\mathrm{d}}{\mathrm{d} t} x=f(x)$ is called globally attractive with respect to a compact set $\mathcal{C}$ if $\lim _{t \rightarrow \infty}\left\|\varphi_{f}\left(t, x_{0}\right)\right\|_{\mathcal{C}}=0$ holds for all $x_{0} \in \mathbb{R}^{n}$.

Next, we define two classes of comparison functions which are used to establish convergence properties of the system.

Definition 2 (Comparison functions) A function $\chi_{c}$ : $\mathbb{R}_{\geq 0} \rightarrow \mathbb{R}_{\geq 0}$ is of class $\mathscr{K}$ if it is continuous, strictly increasing and $\chi_{c}(0)=0$; it is of class $\mathscr{K}_{\infty}$ if it is a $\mathscr{K}$ function and $\chi_{c}(s) \rightarrow \infty$ as $s \rightarrow \infty$.

Finally, the following Theorem provides a Lyapunov characterization of attractivity with respect to a set.

Theorem 1 (Lyapunov function) Consider a continuously differentiable vector field $f: \mathbb{R}^{n} \rightarrow \mathbb{R}^{n}$, a compact set $\mathcal{C} \subseteq \mathbb{R}^{n}$, and a continuously differentiable function $\mathcal{V}$ : $\mathbb{R}^{n} \rightarrow \mathbb{R}_{>0}$. The dynamical system $\frac{\mathrm{d}}{\mathrm{d} t} x=f(x)$ is globally attractive with respect to $\mathcal{C}$ if there exist $\chi_{1} \in \mathscr{K}_{\infty}$ and $\chi_{2} \in \mathscr{K}$ such that for all $x \in \mathbb{R}^{n}$ it holds that

$$
\begin{aligned}
\chi_{1}\left(\|x\|_{\mathcal{C}}\right) & \leq \mathcal{V}(x) \\
\frac{\mathrm{d}}{\mathrm{d} t} \mathcal{V}(x):=\frac{\partial \mathcal{V}}{\partial x} f(x) & \leq-\chi_{2}\left(\|x\|_{\mathcal{C}}\right) .
\end{aligned}
$$

The proof follows from standard arguments (see [15, Sec. 25]) and is omitted for reasons of space.

\section{STABILITY ANALYSIS USING SINGULAR PERTURBATION THEORY}

In Section II we introduced the control objective and proposed a control law that admits a decentralized implementation. We will now analyze the closed-loop system and provide sufficient conditions for attractivity of synchronous solutions which satisfy the objectives (7) to (10). To do so, we will use ideas from singular perturbation analysis following similar steps to [16, Sec. 11.5], but consider convergence with respect to a set of steady states instead of the origin. This will then allow us to prove the main result of the paper: a bound for the control parameters (gains) such that the system dynamics (12) converges to a set of steadystates. Moreover, we show that the only steady-state that does not satisfy the control objectives presented in Section II-B is unstable.

\section{A. Dynamics and control objectives in a rotating frame}

Since $\mathcal{R}(\theta)$ commutes with $\mathcal{K}, \mathcal{B}$ and $\Phi(v)=\Phi(\underline{v})$, the dynamics (12) in the rotating coordinates (3) become

$$
\begin{aligned}
\frac{\mathrm{d}}{\mathrm{d} t} v & =\eta(\mathcal{K} v-\mathcal{R}(\kappa) \mathcal{B} i+\alpha \Phi(v) v)=: f_{v}(v, i), \\
\frac{\mathrm{d}}{\mathrm{d} t} i & =L_{T}^{-1}\left(-R_{T} i-L_{T} \mathcal{J}_{M} \omega_{0} i+\mathcal{B}^{\top} v\right)=: f_{i}(v, i) .
\end{aligned}
$$

Moreover, we let $x=(v, i) \in \mathbb{R}^{n}$ with $n=2 N+2 M$ and denote the dynamics of the overall system by $\frac{\mathrm{d}}{\mathrm{d} t} x=f(x)$ with $f(x)=\left(f_{v}(v, i), f_{i}(v, i)\right)$. To formalize the control objectives we define the sets

$$
\begin{aligned}
& \mathcal{S}=\left\{v \in \mathbb{R}^{2 N} \mid v_{k}=R\left(\theta_{k 1}^{\star}\right) v_{1}, \forall k \in \mathcal{N} \backslash\{1\}\right\}, \\
& \mathcal{A}=\left\{v \in \mathbb{R}^{2 N} \mid\left\|v_{k}\right\|=v^{\star}, \forall k \in \mathcal{N}\right\},
\end{aligned}
$$

as well as the target set

$$
\mathcal{T}:=\left\{x \in \mathbb{R}^{n} \mid x=(v, i), v \in \mathcal{S} \cap \mathcal{A}, i=i^{s}(v)\right\} .
$$

Moreover, we define the set $\mathcal{T}_{0}$ :

$$
\mathcal{T}_{0}:=\mathcal{T} \cup\left\{\mathbb{O}_{n}\right\} .
$$

Note that all elements of $\mathcal{T}_{0}$ are equilibria for the dynamics in the rotating reference frame (15). Therefore, in the static frame, they correspond to synchronous sinusoidal trajectories with frequency $\omega_{0}$. Furthermore, by the very definition of the sets $\mathcal{S}, \mathcal{A}$, and $\mathcal{T}$ they also satisfy all control objectives introduced in Section II-B.

\section{B. Main result}

We require the following stability condition on the network parameters, steady-state angles, and control gains:

Condition 1 (Stability Condition) The graph $\mathcal{G}$, the steadystate angles $\theta_{j k}^{\star}$ with $\bar{\theta}^{\star}:=\max _{(j, k) \in \mathcal{E}} \theta_{j k}^{\star}$, and the gain $\alpha$ are such that the following inequality holds

$$
\max _{1 \leq k \leq n} \sum_{j:(j, k) \in \mathcal{E}}\left\|Y_{j k}\right\|\left|\sin \left(\theta_{j k}^{\star}\right)\right|+\alpha<\frac{1+\cos \left(\bar{\theta}^{\star}\right)}{2} \lambda_{2}(L),
$$

where $\lambda_{2}(L)$ denotes the second smallest eigenvalue of the Laplacian $L$ and we define $c\left(\mathcal{G}, \theta_{j k}^{\star}, \alpha\right):=\frac{1}{2}(1+$ $\left.\cos \left(\bar{\theta}^{\star}\right)\right) \lambda_{2}(L)-\max _{1 \leq k \leq n} \sum_{j:(j, k) \in \mathcal{E}}|| Y_{j k} \||| \sin \left(\theta_{j k}^{\star}\right) \mid-\alpha$. Moreover, given $\bar{Y}:=\max _{(j, k) \in \mathcal{E}}\left\|Y_{j k}\right\|$, $\eta$ satisfies

$$
\eta<\frac{c\left(\mathcal{G}, \theta_{j k}^{\star}, \alpha\right)}{\rho \bar{Y}\|\mathcal{B}\|^{2}\left(c\left(\mathcal{G}, \theta_{j k}^{\star}, \alpha\right)+5\|\mathcal{K}-\mathcal{L}\|\right)} .
$$

We can now state the main result of the manuscript.

Theorem 2 (Convergence of the closed loop) Consider steady-state angles $\theta_{j k}^{\star}$ and control gains $\alpha$ and $\eta$ such that Condition 1 holds. Then, the dynamics (15) are globally attractive with respect to $\mathcal{T}_{0}$. Moreover, the origin $\mathbb{O}_{n}$ is an unstable equilibrium. 
Theorem 2 guarantees global attractivity of the union of the desired set of equilibria (corresponding to harmonic solution with desired power flows) and the origin (trivial solution with zero voltages and currents) and establishes that the origin is an unstable equilibrium of the system. The rest of this section is devoted to proving Theorem 2.

\section{Singular perturbation theory}

In the following we will apply tools from singular perturbation theory to explicitly construct a Lyapunov function that establishes convergence of the dynamics (15) to the set $\mathcal{T}_{0}$ and allows us to show that the origin $\mathbb{O}_{n}$ is an unstable equilibrium. By replacing the dynamics (15b) of the transmission lines with its steady-state map $i^{s}(v)$, we obtain the reduced system

$$
\frac{\mathrm{d}}{\mathrm{d} t} v=f_{v}\left(v, i^{s}(v)\right)=\eta[(\mathcal{K}-\mathcal{L}) v+\alpha \Phi(v)] v
$$

which can be interpreted as the voltage dynamics under the assumption that the line currents are at their rotational steadystate and therefore $i_{o}=\mathcal{Y} v=R(\kappa)^{\top} \mathcal{L} v$. Finally, we denote the difference between the line currents and their steady state value as $y=i-i^{s}(v)$ and define the boundary-layer system

$$
\left.\frac{\mathrm{d} y}{\mathrm{~d} t}\right|_{\frac{\mathrm{d}}{\mathrm{d} t} v=0}=f_{i}\left(v, y+i^{s}(v)\right)
$$

where $v$ is treated as a constant. We now follow a similar approach to [16] to obtain conditions on the convergence of the full system (15) based on the independent study of the convergence of (18) and (19). Differently from [16] we address attractivity with respect to the set $\mathcal{T}_{0}$ and not a single equilibrium.

\section{Lyapunov function for the reduced system}

Let us begin by considering the reduced system (18). Given the steady-state angles $\theta_{k 1}^{\star}$ with $\theta_{k 1}^{\star} \in[0, \pi / 2]$, let us define the matrix $S$ as $S:=\left[R\left(\theta_{11}^{\star}\right)^{\top} \ldots R\left(\theta_{1 N}^{\star}\right)^{\top}\right]^{\top}$ and $\mathbb{I}:=\left[I_{2} \ldots I_{2}\right]^{\top}=\mathbb{1} \otimes I_{2}$. We further denote by $P_{S}:=\left(I_{2 N}-\frac{1}{n} S S^{\top}\right)$ the projector onto the span of $S$. We now define the Lyapunov function candidate $V: \mathbb{R}^{n} \rightarrow \mathbb{R}_{\geq 0}$ for the reduced system as

$$
V(v):=\frac{1}{2} v^{\top} P_{S} v+\eta \alpha \alpha_{1} \sum_{k=1}^{N}\left(\frac{v^{\star 2}-\left\|v_{k}\right\|^{2}}{v^{\star}}\right)^{2},
$$

where $\alpha$ is the voltage controller gain and the positive constant $\alpha_{1}$ is given by

$$
\alpha_{1}:=\frac{c\left(\mathcal{G}, \theta_{i k}^{\star}, \alpha\right)}{5 \eta\|\mathcal{K}-\mathcal{L}\|^{2}} .
$$

We also define the function $\psi: \mathbb{R}^{2 N} \rightarrow \mathbb{R}_{\geq 0}$ as

$$
\psi(v):=\eta\left(\|\mathcal{K}-\mathcal{L}\|\|v\|_{\mathcal{S}}+\alpha\|\Phi(v) v\|\right) .
$$

In the following Theorem, we show the function $V$ is a Lyapunov function for the reduced system (18).

Proposition 1 (Convergence of the reduced system) Consider $\theta_{j k}^{\star}, \alpha$, and $\eta$ such that Condition 1 holds. For all $v \in \mathbb{R}^{2 N}$ the derivative of $V$ along the trajectories of the reduced system (18) satisfies

$$
\frac{\mathrm{d}}{\mathrm{d} t} V(v):=\frac{\partial V}{\partial v} f_{v}\left(v, i^{s}(v)\right) \leq-\alpha_{1} \psi(v)^{2} .
$$

Proof: We can write the derivative of $V(\cdot)$ along the trajectories of the reduced system (18) as

$$
\begin{aligned}
\frac{\mathrm{d}}{\mathrm{d} t} V(v) & =\eta v^{\top} P_{S}((\mathcal{K}-\mathcal{L}) v+\alpha \Phi(v) v) \\
& -2 \eta^{2} \alpha \alpha_{1} v^{\top} \Phi(v)((\mathcal{K}-\mathcal{L}) v+\alpha \Phi(v) v)
\end{aligned}
$$

Next, following the approach used in [9, Propositon 6] it can be shown that

$$
v^{\top} P_{S} \Phi(v) v \leq v^{\top} P_{S} v=\|v\|_{\mathcal{S}}^{2}
$$

Under Assumption 1, using the same techniques as in [9, Proposition 7] and (25), we can bound (24) as

$$
\begin{aligned}
\frac{\mathrm{d}}{\mathrm{d} t} V(v) & \leq-\eta c\left(\mathcal{G}, \theta_{i k}^{\star}, \alpha\right)\|v\|_{\mathcal{S}}^{2} \\
& -2 \eta^{2} \alpha \alpha_{1} v^{\top} \Phi(v)((\mathcal{K}-\mathcal{L}) v+\alpha \Phi(v) v) .
\end{aligned}
$$

Exploiting the fact that $\mathcal{K}-\mathcal{L}=(\mathcal{K}-\mathcal{L}) P_{S}$, we can further bound (26) as

$$
\begin{aligned}
\frac{\mathrm{d}}{\mathrm{d} t} V(v) \leq & -\eta c\left(\mathcal{G}, \theta_{i k}^{\star}, \alpha\right)\|v\|_{\mathcal{S}}^{2}-2 \eta^{2} \alpha^{2} \alpha_{1}\|\Phi(v) v\|^{2} \\
& +2 \eta^{2} \alpha \alpha_{1}\|\Phi(v) v\|\|\mathcal{K}-\mathcal{L}\|\|v\|_{S} .
\end{aligned}
$$

In order to prove that $\frac{\mathrm{d}}{\mathrm{d} t} V(v)$ satisfies (23), we need to show that the inequality

$$
\begin{aligned}
& -\eta c\left(\mathcal{G}, \theta_{i k}^{\star}, \alpha\right)\|v\|_{\mathcal{S}}^{2}-2 \eta^{2} \alpha^{2} \alpha_{1}\|\Phi(v) v\|^{2} \\
& +2 \eta^{2} \alpha \alpha_{1}\|\Phi(v) v\|\|\mathcal{K}-\mathcal{L}\|\|v\|_{S} \\
& \leq-\alpha_{1} \eta^{2}\left(\|\mathcal{K}-\mathcal{L}\|\|v\|_{\mathcal{S}}+\alpha\|\Phi(v) v\|\right)^{2}
\end{aligned}
$$

holds for $\alpha_{1}$ according to (21). By matching the coefficients of the r.h.s and the 1.h.s., we can rewrite (27) as

$$
\left[\begin{array}{c}
\|v\|_{\mathcal{S}} \\
\|\Phi(v) v\|
\end{array}\right]^{\top} Q\left[\begin{array}{c}
\|v\|_{\mathcal{S}} \\
\|\Phi(v) v\|
\end{array}\right] \geq 0, \quad \forall v \in \mathbb{R}^{2 N}
$$

with the matrix $Q$ defined as

$Q:=\left[\begin{array}{cc}\eta c\left(\mathcal{G}, \theta_{i k}^{\star}, \alpha\right)-\alpha_{1} \eta^{2}\|\mathcal{K}-\mathcal{L}\|^{2} & -2 \alpha_{1} \alpha \eta^{2}\|\mathcal{K}-\mathcal{L}\| \\ \star & \eta^{2} \alpha^{2} \alpha_{1}\end{array}\right]$.

Using the Schur complement and $\alpha_{1}>0$ it follows that $Q \succ 0$ if $\eta c-5 \alpha_{1} \eta^{2}\|\mathcal{K}-\mathcal{L}\|^{2} \geq 0$. Thus, $\alpha_{1}$ defined in (21) satisfies (28) and the proposition directly follows.

\section{E. Lyapunov function for the Boundary layer system}

In this section, we establish exponential convergence of the boundary layer system (19). To this end, the Lyapunov function candidate $W: \mathbb{R}^{2 M} \rightarrow \mathbb{R}_{\geq 0}$ is defined as

$$
W(y):=\frac{\rho}{2} y^{\top} y
$$

Proposition 2 (Convergence of the boundary layer system) For every fixed $v \in \mathbb{R}^{2 N}$ and all $y \in \mathbb{R}^{2 M}$, the derivative of $W(y)$ along the trajectories of (19) satisfies

$$
\frac{\mathrm{d}}{\mathrm{d} t} W(y):=\frac{\partial W}{\partial y} f_{i}\left(v, y+i^{s}(v)\right) \leq-\alpha_{2}\|y\|^{2},
$$


where $\alpha_{2}:=\sqrt{1+\omega_{0}^{2} \rho^{2}} \cos (\kappa)$.

Proof: Using Assumption 1 and $\mathcal{R}_{M}(\kappa)$ := $I_{M} \otimes R(\kappa)$, it can be verified that $f_{i}\left(v, y+i^{s}(v)\right)=$ $-\sqrt{\frac{1}{\rho^{2}}+\omega_{0}^{2}} \mathcal{R}_{M}(\kappa) y$. This results in

$\frac{\mathrm{d}}{\mathrm{d} t} W(y)=\rho y^{\top} f_{i}\left(v, y+i^{s}(v)\right)=-\rho \sqrt{\frac{1}{\rho^{2}}+\omega_{0}^{2}} y^{\top} \mathcal{R}_{M}(\kappa) y$, and the proposition immediately follows.

\section{F. Proof of the main result}

In Sections IV-D and IV-E we established that the Lyapunov functions for the reduced system (18) and the boundary layer system (19) respectively are decreasing. In this section, we use these results to construct a Lyapunov function candidate for the overall system (15). The reduced system (18) describes the voltage dynamics assuming the currents are in steady-state and the boundary layer system describes the dynamics of the transmission line currents under the assumption that the voltages are constant (in the rotating reference frame). The following propositions aim at bounding the increase of the Lyapunov functions resulting from considering the full dynamics (15).

Proposition 3 Let $\beta_{1}=\frac{\|\mathcal{B}\|}{\|\mathcal{K}-\mathcal{L}\|}$. For all $v \in \mathbb{R}^{2 N}$ and $y \in$ $\mathbb{R}^{2 M}$ it holds that

$$
\frac{\partial V}{\partial v}\left[f_{v}\left(v, y+i^{s}(v)\right)-f_{v}\left(v, i^{s}(v)\right)\right] \leq \beta_{1} \psi(v)\|y\| .
$$

Proof: The function $f_{v}(v, i)$ introduced in (15a), is separable in its two arguments and linear in $i$. Hence,

$$
f_{v}\left(v, y+i^{s}(v)\right)-f_{v}\left(v, i^{s}(v)\right)=f_{v}(0, y)=\eta R(\kappa) \mathcal{B} y .
$$

It directly follows that

$$
\begin{aligned}
& \frac{\partial V}{\partial v}\left[f_{v}\left(v, y+i^{s}(v)\right)-f_{v}\left(v, i^{s}(v)\right)\right] \leq \\
& =\left(\eta v^{\top} P_{S}+2 \alpha \alpha_{1} \eta^{2} v^{\top} \Phi(v)\right) R(\kappa) \mathcal{B} y \\
& \leq\left(\eta\|\mathcal{B}\|\|v\|_{\mathcal{S}}+2 \alpha \alpha_{1} \eta^{2}\|\mathcal{B}\|\|\phi(v) v\|\right)\|y\| \leq \beta_{1} \psi(v)\|y\|,
\end{aligned}
$$

with $\beta_{1}:=\max \left(\frac{\|\mathcal{B}\|}{\|\mathcal{K}-\mathcal{L}\|}, 2 \alpha_{1} \eta\|\mathcal{B}\|\right)$. From (21) and the fact that $c\left(\mathcal{G}, \theta_{i k}^{\star}, \alpha\right)<\|\mathcal{K}-\mathcal{L}\|$ we conclude that $2 \alpha_{1} \eta\|\mathcal{B}\| \leq$ $\frac{2 c\left(\mathcal{G}, \theta_{i k}^{*}, \alpha\right)\|\mathcal{B}\|}{5\|\mathcal{K}-\mathcal{L}\|^{2}} \leq \frac{\|\mathcal{B}\|}{\|\mathcal{K}-\mathcal{L}\|}$. Therefore, $\beta_{1}=\frac{\|\mathcal{B}\|}{\|\mathcal{K}-\mathcal{L}\|}$, and the proof is complete.

Proposition 4 Consider $\beta_{2}:=\rho\left(\sqrt{\frac{1}{\rho^{2}}+\omega_{0}^{2}}\right)^{-1}\left\|L_{T}^{-1} \mathcal{B}^{\top}\right\|$ and $\gamma:=\eta \rho\left(\sqrt{\frac{1}{\rho^{2}}+\omega_{0}^{2}}\right)^{-1}\left\|L_{T}^{-1} \mathcal{B}^{\top} \mathcal{B}\right\|$. For all $v \in \mathbb{R}^{2 N}$ and $y \in \mathbb{R}^{2 M}$ it holds that

$$
\left[\frac{\partial W}{\partial v}-\frac{\partial W}{\partial y} \frac{\partial i^{s}}{\partial v}\right] f_{v}\left(v, y+i^{s}(v)\right) \leq \beta_{2} \psi(v)\|y\|+\gamma\|y\|^{2} .
$$

Proof: Using again the linearity of $f_{v}(v, i)$ in $i$ and (5) we can write

$$
\begin{aligned}
& {\left[\frac{\partial W}{\partial v}-\frac{\partial W}{\partial y} \frac{\partial i^{s}}{\partial v}\right] f_{v}\left(v, y+i^{s}(v)\right)} \\
& =\frac{-\rho}{\sqrt{\frac{1}{\rho^{2}}+\omega_{0}^{2}}} y^{\top} R(\kappa)^{\top} L_{T}^{-1} \mathcal{B}^{\top}\left(f_{v}\left(v, i^{s}(v)\right)+f_{v}(0, y)\right)
\end{aligned}
$$

We can bound

$$
\left\|f_{v}\left(v, i^{s}(v)\right)\right\| \leq \eta\|\mathcal{K}-\mathcal{L}\|\|v\|_{\mathcal{S}}+\eta \alpha\|\Phi(v) v\|=\psi(v) .
$$

The result follows by combining (31), (32) and (30). Using $d=\frac{\beta_{1}}{\beta_{1}+\beta_{2}}>0$ and the functions $V(v)$ and $W(y)$ introduced in (20) and (29), we define the Lyapunov function candidate $\nu: \mathbb{R}^{n} \rightarrow \mathbb{R}_{\geq 0}$ for the overall system (15) as

$$
\nu(x):=d W\left(i-i^{s}(v)\right)+(1-d) V(v) .
$$

Finally, we can bound the decrease of $\nu$ along the trajectories of the system (15).

Proposition 5 Under Assumption 1, there exist a function $\chi_{2} \in \mathscr{K}$ such that the derivative of $\nu$ along the trajectories of the system (15) satisfies

$$
\frac{\mathrm{d}}{\mathrm{d} t} \nu:=\frac{\partial \nu}{\partial v} f_{v}(v, i)+\frac{\partial \nu}{\partial v} f_{i}(v, i) \leq-\chi_{2}\left(\|x\|_{\mathcal{T}_{0}}\right)
$$

Proof: Using Propositions 1 - 4 and following similar steps to [16] the derivative of $\nu$ along the trajectories of the full system (15) satisfies the inequality

$$
\frac{\mathrm{d}}{\mathrm{d} t} \nu \leq-\left[\begin{array}{c}
\psi(v) \\
\|y\|
\end{array}\right]^{\top}\left[\begin{array}{cc}
(1-d) \alpha_{1} & -\frac{(1-d) \beta_{1}+d \beta_{2}}{2} \\
\star & d\left(\alpha_{2}-\gamma\right)
\end{array}\right]\left[\begin{array}{c}
\psi(v) \\
\|y\|
\end{array}\right],
$$

where $y=i-i^{s}(v)$ and the quadratic form on the r.h.s. is positive definite if

$$
1<\frac{\alpha_{1} \alpha_{2}}{\alpha_{1} \gamma+\beta_{1} \beta_{2}} .
$$

Using the expressions for $\alpha_{1}, \alpha_{2}, \beta_{1}, \beta_{2}$ and $\gamma$ derived in Propositions $1-4$, condition (35) is satisfied if

$$
\eta<\frac{c\left(\mathcal{G}, \theta_{i k}^{\star}, \alpha\right)\left(\frac{1}{\rho^{2}}+\omega_{0}^{2}\right) \cos (\kappa)}{\left\|L_{T}^{-1} \mathcal{B}^{\top}\right\|\|\mathcal{B}\|\left(c\left(\mathcal{G}, \theta_{i k}^{\star}, \alpha\right)+5\|\mathcal{K}-\mathcal{L}\|\right)} .
$$

Noting that $\cos (\kappa)=r_{i j} /\left(\sqrt{r_{i j}^{2}+\omega_{0}^{2} \ell_{i j}^{2}}\right)$, it can be verified that (36) holds under Condition 1 and it follows that $\frac{\mathrm{d}}{\mathrm{d} t} \nu(x)<0$ for all $(v, i) \notin \mathcal{T}_{0}$. Moreover, because $\mathcal{T}_{0}$ is compact, and $\psi(v)$ is positive definite and radially unbounded with respect to $(\mathcal{S} \cap \mathcal{A}) \cup\left\{\mathbb{0}_{2 N}\right\}$, the steps in [15, p. 98] can be used to show that there exists a function $\chi_{2} \in \mathscr{K}$ such that (34) holds.

We are now ready to prove Theorem 2 .

\section{Proof of Theorem 2:}

Note that $\nu(x)$ is positive definite with respect to $\mathcal{T}$, i.e., $\nu(x)=0$ for $x \in \mathcal{T}$ and $\nu(x)>0$ otherwise, and radially unbounded with respect to $\mathcal{T}$, i.e., $\|x\|_{\mathcal{T}} \rightarrow \infty$ implies that $\nu(x) \rightarrow \infty$. Moreover $\mathcal{T}$ is a compact set, and, following the steps in [15, p. 98], there exist $\chi_{1}^{\prime} \in \mathscr{K}_{\infty}$ such that $\chi_{1}^{\prime}\left(\|x\|_{\mathcal{T}}\right) \leq \nu(x)$ for all $x \in \mathbb{R}^{2 N}$. Because $\mathcal{T} \subset \mathcal{T}_{0}$ there exists $\chi_{1} \in \mathscr{K}_{\infty}$ such that $\chi_{1}\left(\|x\|_{\mathcal{T}_{0}}\right) \leq \chi_{1}^{\prime}\left(\|x\|_{\mathcal{T}}\right) \leq \nu(x)$ for all $x \in \mathbb{R}^{n}$. Moreover, it follows from Proposition 5 that there exists $\chi_{2} \in \mathscr{K}$ such that $\frac{\mathrm{d}}{\mathrm{d} t} \nu \leq-\chi_{2}\left(\|x\|_{\mathcal{T}_{0}}\right)$ holds for all $x \in \mathbb{R}^{n}$. It follows from Theorem 1 that the dynamics (15) are globally attractive with respect to $\mathcal{T}_{0}$.

To show that $\mathbb{O}_{n}$ is an unstable equilibrium, let $\varepsilon^{\prime}:=\frac{1}{2} v^{\star}$, pick any $v_{0}^{\prime} \in \mathcal{S} \backslash \mathbb{D}_{2 N}$ such that $\left\|v_{0}^{\prime}\right\|=\varepsilon^{\prime}$, and let $x_{0}^{\prime}:=\left(v_{0}^{\prime}, h\left(v_{0}^{\prime}\right)\right)$. For any constant $c_{0} \in \mathbb{R}_{(0,1)}$ it holds 
that $\nu\left(c_{0} x_{0}^{\prime}\right)<\nu\left(\mathbb{O}_{n}\right)$. Moreover, Proposition 5 implies that $\frac{\mathrm{d}}{\mathrm{d} t} \nu(x)<0$ for all $x \notin \mathcal{T}_{0}$. It follows that the trajectories starting at $c_{0} x_{0}^{\prime}$ cannot converge to the origin, so they must converge to $\mathcal{T}$. Therefore, no matter how small $c_{0}$ is chosen, there exist a time $t^{\prime}$ such that $\left\|\varphi_{f}\left(t^{\prime}, c_{0} x_{0}^{\prime}\right)\right\|>\epsilon^{\prime}$, and it follows that the origin is an unstable equilibrium of (15).

\section{POWER SySTEMS TEST-CASE}

In this section, we demonstrate the decentralized synchronizing controller with a power systems test-case. We consider three inverters connected by resistive-inductive lines (two lines of $25 \mathrm{~km}$ and one of $125 \mathrm{~km}$ from inverters 1 and 2). The grid base power is $1 \mathrm{GW}$, the base voltage $320 \mathrm{kV}$. The line resistance is $0.03 \mathrm{Ohm} / \mathrm{km}$ and the line reactance is $0.3 \mathrm{Ohm} / \mathrm{km}$ (at $50 \mathrm{~Hz}$ ). Therefore, the inductance/resistance ratio $\rho$ of the transmission lines is given by $\omega_{0} \rho=10$. The set-points are $\omega_{0}=2 \pi 50, v^{\star}=1$, and the steady-state angles are $\theta_{21}^{\star}=\theta_{31}^{\star}=1^{\circ}$. Each inverter implements the control law (11). We chose $\alpha=5$, and, from Theorem 2, we obtain a guarantee of synchronization for $\eta \leq 2.9 \cdot 10^{-4}$. Figure 1 shows a simulation with $\eta=2.8 \cdot 10^{-4}$ where the controller synchronizes the network. In Figure 2 we see that the states do not converge to the desired operating point for $\eta=2.8 \cdot 10^{-3}$.
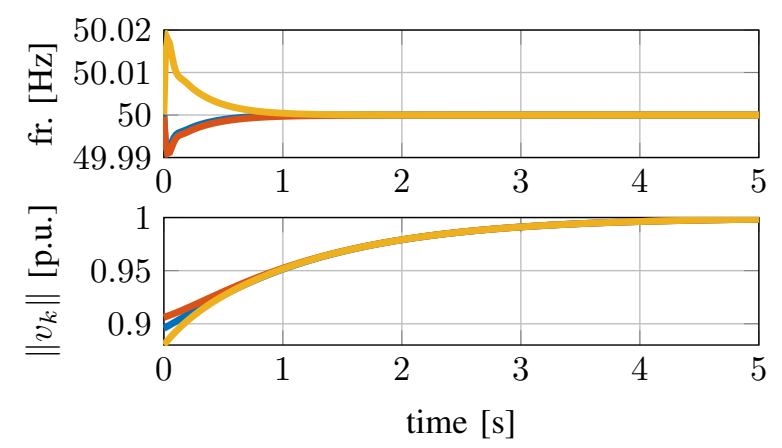

Fig. 1. As predicted by Theorem 2, for $\eta=2.8 \cdot 10^{-4}$, the system synchronizes to the desired solution, i.e., the system converges to $\mathcal{T}$. Note that the bounds predicted by Theorem 2 are only sufficient and thus conservative.
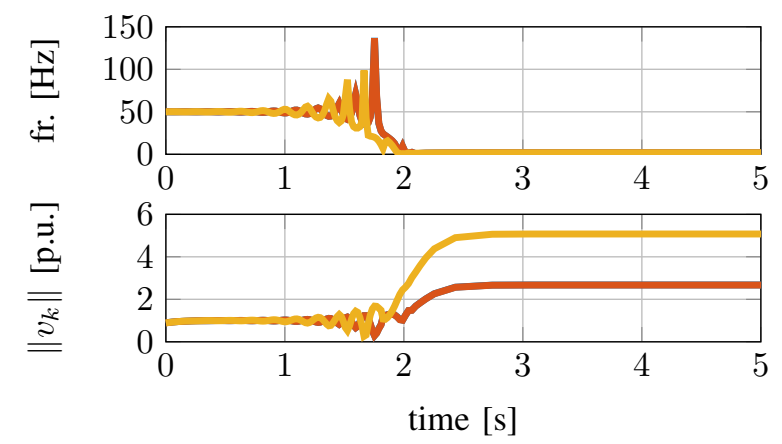

Fig. 2. With $\eta=2.8 \cdot 10^{-3}$, i.e., larger than the bound from Theorem 2 , the system converges to a limit cycle which is not contained in $\mathcal{T}$.

\section{CONCLUSION AND OUTLOOK}

In this paper, we analyzed the effect of transmissionline dynamics on the convergence properties of a network of grid-forming power inverters using dispatchable virtual oscillator control (dVOC). While dVOC ensures almost global asymptotic stability when a quasi-steady-state network model is used, this result no longer holds when considering the dynamics of transmission lines. Using tools from singular perturbation theory, we obtained explicit bounds on the controller gains that guarantee global attractivity of the desired steady-state behavior as well as the origin. In a separate argument, we showed that the only undesirable steady-state, i.e., origin, is an unstable equilibrium of the system. The results of this article provide a first step towards establishing conditions under which dVOC ensures almost global asymptotic stability of an inverter based AC power system with respect to a pre-specified solution of the AC power-flow equations.

\section{REFERENCES}

[1] M. C. Chandorkar, D. M. Divan, and R. Adapa, "Control of parallel connected inverters in standalone AC supply systems," IEEE Transactions on Industry Applications, vol. 29, no. 1, pp. 136-143, 1993.

[2] V. Mariani, F. Vasca, J. C. Vásquez, and J. M. Guerrero, "Model order reductions for stability analysis of islanded microgrids with droop control," IEEE Transactions on Industrial Electronics, vol. 62, no. 7, pp. 4344-4354, 2015.

[3] B. B. Johnson, S. V. Dhople, A. O. Hamadeh, and P. T. Krein, "Synchronization of parallel single-phase inverters with virtual oscillator control," IEEE Transactions on Power Electronics, vol. 29, no. 11, pp. 6124-6138, 2014.

[4] J. W. Simpson-Porco, F. Dörfler, and F. Bullo, "Voltage stabilization in microgrids via quadratic droop control," IEEE Transactions on Automatic Control, vol. 62, no. 3, pp. 1239-1253, 2017.

[5] M. Sinha, F. Dörfler, B. Johnson, and S. Dhople, "Uncovering droop control laws embedded within the nonlinear dynamics of van der pol oscillators," IEEE Transactions on Control of Network Systems, vol. 4, no. 2, pp. 347-358, 2017.

[6] L. Tôrres, J. Hespanha, and J. Moehlis, "Synchronization of oscillators coupled through a network with dynamics: A constructive approach with applications to the parallel operation of voltage power supplies," 2013, submitted.

[7] B. B. Johnson, M. Sinha, N. G. Ainsworth, F. Dörfler, and S. V. Dhople, "Synthesizing virtual oscillators to control islanded inverters," IEEE Transactions on Power Electronics, vol. 31, no. 8, pp. 60026015, 2016.

[8] M. Colombino, D. Groß, and F. Dörfler, "Global phase and voltage synchronization for power inverters: A decentralized consensusinspired approach," in IEEE Conference on Decision and Control, 2017, pp. 5690-5695.

[9] M. Colombino, D. Groß, J.-S. Brouillon, and F. Dörfler, "Global phase and magnitude synchronization of coupled oscillators with application to the control of grid-forming power inverters," arXiv preprint arXiv:1710.00694, 2017.

[10] S. Curi, D. Groß, and F. Dörfler, "Control of low-inertia power grids: A model reduction approach," in IEEE Conference on Decision and Control, 2017, pp. 5708-5713.

[11] P. Vorobev, P.-H. Huang, M. Al Hosani, J. L. Kirtley, and K. Turitsyn, "High-fidelity model order reduction for microgrids stability assessment," IEEE Transactions on Power Systems, 2017.

[12] P. Vorobev, P. H. Huang, M. A. Hosani, J. L. Kirtley, and K. Turitsyn, "A framework for development of universal rules for microgrids stability and control," in IEEE Conference on Decision and Control, 2017, pp. 5125-5130.

[13] D. Groß, M. Colombino, J.-S. Brouillon, and F. Dörfler, "The effect of transmission-line dynamics on grid-forming dispatchable virtual oscillator control," arXiv preprint arXiv:1802.08881, 2018.

[14] E. Clarke, Circuit analysis of AC power systems. Wiley, 1943, vol. 1.

[15] W. Hahn, Stability of motion. Springer, 1967.

[16] H. K. Khalil, Nonlinear systems, 3rd ed. Prentice Hall, 2002. 